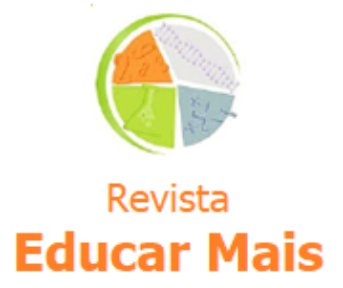

\section{Projeto Robótica Pedagógica: o resgate do PROUCA para o Ensino de Ciências - na educação 4.0}

\author{
Pedagogical Robotic project: the rescue of PROUCA for the \\ Teaching of Sciences - in education 4.0
}

David Gentil de Oliveira ${ }^{1}$; Wellington da Silva Fonseca ${ }^{1}$

\begin{abstract}
RESUMO
O presente artigo vem demonstrar a importância da Robótica Pedagógica como forma de ensino nos anos finais do Ensino Fundamental, por meio do resgate do Programa Um Computador por Aluno (Prouca) para a Robótica Pedagógica, as plataformas Arduino e Ardublockly, de acordo com a BNCC - Base Nacional Comum Curricular junto ao Ensino de Ciências - Física. Com isso, desenvolveu-se uma análise quantitativa, a partir de projeto de oficinas e treinamentos de introdução à robótica pedagógica na escola municipal Major Cornélio Peixoto no município de Santo Antônio do Tauá no estado do Pará, possibilitando aos professores e alunos a oportunidade de ampliar as discussões das teorias estudadas em sala de aula, com apoio do Laboratório de Engenhocas da Universidade Federal do Pará (UFPA). observou-se, que a utilização da Robótica Pedagógica deve ser incentivada, planejada e desenvolvida considerando os aspectos de aprendizagem, interagindo de forma consciente com os alunos, propiciando um despertar ativo e dedicado, o que irá refletir positivamente no desenvolvimento crítico do aluno com relação aos seus conhecimentos científicos, estimulando para a formação de futuros engenheiros, e profissionais da área de Ciências Exatas em geral, para que possam utilizar de todo aprendizado adquirido na área de ciência e tecnologia, para assim, ingressarem de forma qualificada no mercado de trabalho.
\end{abstract}

Palavras-chave: Robótica Pedagógica; PROUCA.; Arduino; Ardublockly; BNCC.

\begin{abstract}
This article demonstrates the importance of Pedagogical Robotics as a way of teaching in the final years of Elementary School, through the rescue of the One Computer Per Student (Prouca) Program for Pedagogical Robotics, the Arduino and Ardublockly platforms, BNCC - Base National Curricular Joint with the Teaching of Sciences - Physics. With this, an analysis was developed, through the design of workshops and introductory training in pedagogic robotics in the municipal schools of Major Cornélio Peixoto in the municipality of Santo Antônio do Tauá in the state of Pará, in such a way that they lead teachers and students to opportunity to work and discuss the theory of content given in the classroom, with support from the Laboratory of Engenhocas of the Federal University of Pará (UFPA). From this, it is noticed that the use of Pedagogical Robotics should be encouraged, planned and developed in order to consider the learning aspects, interacting in a conscious way with the students, providing an active and dedicated awakening, which will reflect positively on the critical development of the student in relation to their scientific knowledge, stimulating for the training of future engineers, and professionals in the area of Exact Sciences in general, so that they can use all acquired learning in the area of science and technology, in order to enter in a way qualified in the labor market.
\end{abstract}

Keywords: Pedagogical Robotics; PROUCA; Arduino; Ardublockly; BNCC.

${ }^{1}$ UFPA - Universidade Federal do Pará, Belém/PA - Brasil. 


\section{INTRODUÇÃO}

É notório que o ensino de física e fenômenos relacionados à natureza têm pouca relevância nos anos finais do Ensino Fundamental. Isto porque o maior enfoque é dado às ciências biológicas. Porém é neste momento que a criança deve ter o contato com certos conceitos científicos a fim de despertar o gosto pela ciência. Embora o currículo escolar proponha a importância de ensinar os conteúdos de física, os professores dão pouca relevância aos conhecimentos relacionados a esta. Muitos valorizam a memorização dos alunos, e desse modo, as atividades práticas são meras ocupações para os alunos. Logo, a abordagem conteudista pouco contribui para instigar os discentes a pensarem e tornarem-se críticos, uma vez que o estudo de ciências é primordial para o desenvolvimento da sociedade (CAMPOS et al., 2012).

Logo, é inegável que o uso de novas tecnologias, educação 4.0, são importantes ferramentas no ensino para facilitar aprendizagem, neste sentido o ambiente (escola) deve estar preparado para atender uma clientela, mais exigente, ou seja, as novas gerações, $\mathrm{X}, \mathrm{Z}$ e alpha (TOLEDO; ALBUQUERQUE; MAGALHÃES, 2012).

Sendo assim, este trabalho apresenta uma proposta do Ensino de Ciências utilizando-se da Robótica Pedagógica na Escola E. M. E. F. MAJOR CORNELIO PEIXOTO (INEP: 15047903), Município de santo Antônio do Tauá - Pará, por meio de atividades experimentais visando o aprendizado divertido e prazeroso, além de desenvolver nos alunos habilidades, tais como conclusões, argumentações, fortalecendo também as relações interpessoais, incentivando-os a fazer inferências importantes para seu aprendizado. Fora esses aspectos os discentes são despertados ao contato com a ciência a partir da participação em Olimpíadas Cientíicas, deste modo, acredita-se que o projeto seja uma forma de promoção da alfabetização científica.

Além disso, o artigo tem como objetivo, demonstrar o desenvolvimento do projeto Robótica Pedagógica, os seus avanços, principalmente a aquisição de equipamentos, e investigar a possibilidade da sua utilização como recurso pedagógico para o ensino e a aprendizagem de conceitos físicos nos anos finais do Ensino Fundamental. Para isso, como objetivos específicos, pretendeu-se propor atividades experimentais, utilizando-se os computadores do Programa Um Computador por Aluno (Prouca), as plataformas Arduino e o Ardublockly e, através dos dados apresentados, propor exemplo de atividade para as aulas de Ciências que possam contribuir no desenvolvimento de habilidades nos estudantes.

\section{PROJETO ROBÓTICA PEDAGÓGICA: O REGATE DO PROGRAMA UM COMPUTADOR POR ALUNO (PROUCA)}

O Projeto Robótica Pedagógica nas escolas municipais de Santo Antônio do Tauá no Estado do Pará teve sua implantação pelo programa de Extensão da Universidade Federal do Pará - laboratório de Engenhocas no ano de 2015 (DE OLIVEIRA et al., 2016).

No início do projeto, 42 alunos foram atendidos no contraturno. As contempladas foram a Major Cornélio Peixoto e a Aloysio da Costa Chaves, pertencentes à rede municipal de ensino. Toda implementação do projeto inicialmente foi difícil, principalmente por tratar-se de escolas públicas. Porém o compromisso de um dos professores da Rede Municipal foi essencial para implantação e desenvolvimento do Projeto Robótica Pedagógica. 
Nos anos de 2016 e 2017, o projeto teve como referência o laboratório da escola Major Cornélio Peixoto, já no ano 2018, o professor responsável do Projeto descobriu a existência de computadores (laptops), Programa Um Computador por Aluno (Prouca), nunca utilizados no município. A partir desse momento, nasce uma nova era para o projeto Robótica Pedagógica no município de Santo Antônio do Tauá, passou por mudanças positivas consideráveis, principalmente no que consiste em ampliar as escolas atendidas. E através da parceria com a Secretaria Municipal de Educação cautelou tais equipamentos a fim de serem utilizados nas atividades do Projeto.

\subsection{O Programa Um Computador Por Aluno (Prouca): Breve Descrição}

Em 2007, deu-se início a uma fase pré-piloto do Projeto Um Computador por Aluno (UCA), através do incentivo do Governo Federal. Foram contempladas apenas cinco escolas no país, a qual receberam equipamentos doados por duas empresas, a Intel e a Encore, e também pela Ong OLPC (ROSA, 2017).

Neste mesmo ano, houve a regulamentação do Programa Um Computador por Aluno (Prouca também conhecido como a terceira fase do Projeto UCA). Os estados e municípios puderam comprar os laptops educacionais aderindo a uma ata de registro de preços, cuja empresa foi habilitada a vender até 600 mil equipamentos (quantidade registrada em edital), sendo esta a Positivo, com o seu modelo de laptop Mobo S7 (ROSA, 2017), Figura 1.

Figura 1 - Laptops educacionais - Prouca

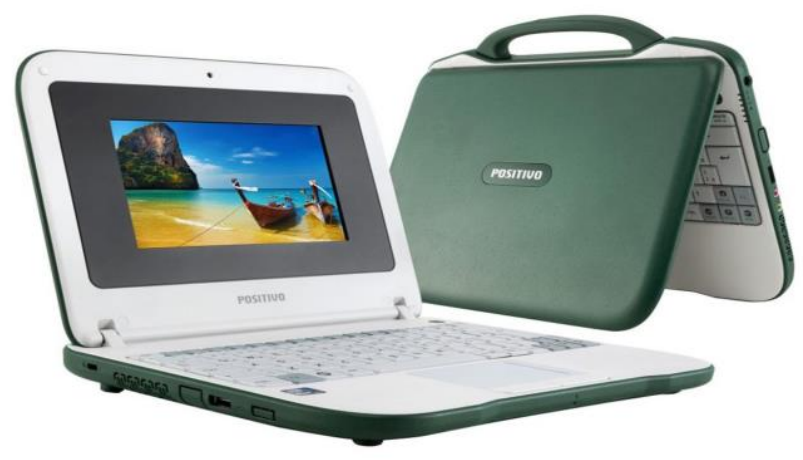

Fonte: FNDE

Segundo Rosa (2017), e as exigências no edital, o laptop Mobo S7, foi distribuído com as seguintes configurações: processador Intel $\circledast$ Atom (TM) com $1.66 \mathrm{GHz}$; memória principal de $1 \mathrm{~GB}$, sendo que, no mínimo 8 MB e no máximo 376 MB são compartilhados com o processador de vídeo - o valor de memória reservado ao processador será subtraído da memória principal, ou seja, quanto mais memória for destinada à qualidade gráfica, menos restará para o sistema operacional e aplicativos, como acontece na maioria dos computadores de custo mais baixo.

Seguindo com a especificação, temos ainda: armazenamento também do tipo cartão de memória, com 4 GB de capacidade; tela de LCD com 7 polegadas, resolução de 800X480; uma câmera VGA, com resolução máxima de $640 \times 480$ (0.3 mega pixel), que grava fotos e vídeos; conexão à rede via cabo (Ethernet) e Wireless; duas portas USB; uma entrada para microfone e outra para fones de ouvido, padrão P2; touchpad com dois botões. O equipamento possui bateria recarregável com duração em torno de quatro horas; alto-falantes embutidos; adaptador bivolt (100 a 240 v) com cabo para recarregar a bateria e alimentação na rede elétrica; com peso aproximado de 1,5 kg. 0 sistema operacional original é o Mandriva Mini (ROSA, 2017). 


\subsection{O PROUCA no Município}

Segundo informação da área técnica de Informática da Secretaria Municipal de Educação de Santo Antônio do Tauá - Pará, o município em 2014 recebeu 3 kits, sendo que cada kit possui 10 laptops Mobo S7, 01 kit destinado a escola E.M.E.F. Martinho Bentes (Inep - 15048179), outro a E.M.E.I.F. Nossa Senhora da Conceição (INEP - 15048330) e por fim um kit destinado a Secretaria Municipal de Educação - SEMED.

No ano de 2018, foi constatada a existência de alguns computadores (laptops) na escola E.M.E.I.F. Nossa Senhora da Conceição, e que estes nunca haviam sido utilizados; o resgate destes equipamentos foi realizado, com o intuito de utilizá-los no Projeto Robótica Pedagógica.

Para a utilização adequada dos laptops Mobo S7, foram realizadas algumas alterações, dentre elas a mudança do cartão de memória, para 32 GB de capacidade de armazenamento e sistema operacional para Windows.

\subsection{Arduino na Educação}

A plataforma o Arduino que utilizam códigos abertos (hardware e software), foi desenvolvida no intuito de colaborar no ensino, e o artigo pretende apresentar a plataforma como ferramenta pedagógica para a próxima o ensino regular ao cotidiano do discente (DE OLIVEIRA; FONSECA, 2018; SOUZA et al., 2011).

Figura 2 - Arduino.

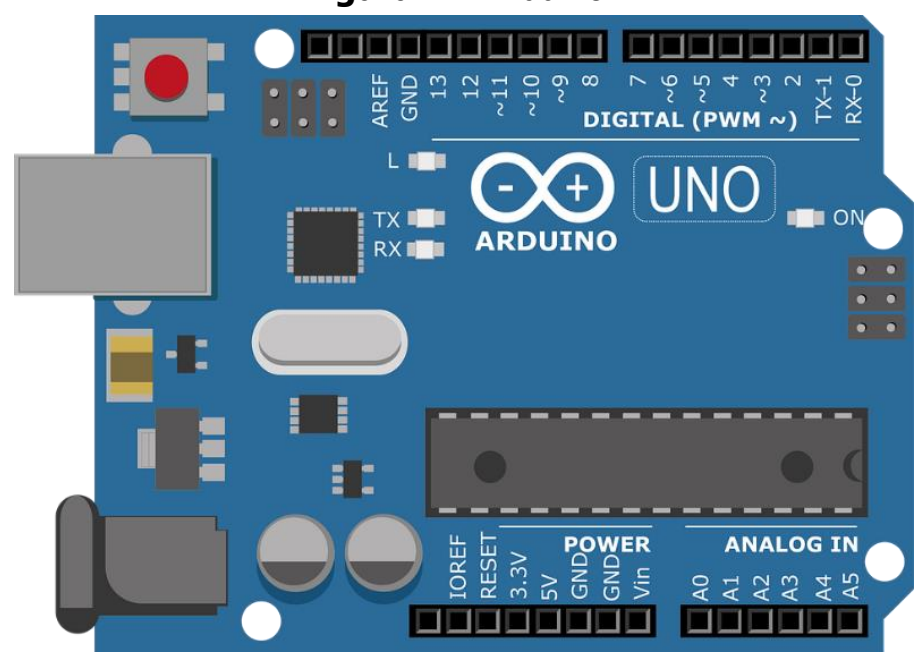

Fonte: https://www.tinkercad.com

\subsection{Ardublockly na Educação}

A plataforma Ardublockly é um programa de edição visual para o Arduino que permite programar através de blocos, facilitando desta forma o ensino-aprendizagem. O software pode ser baixado gratuitamente do site Ardublockly Visual Programming for Arduino: https://ardublockly.embeddedlog.com/index.html (DE OLIVEIRA; FONSECA, 2018). 
Figura 3 - Ardublockly.

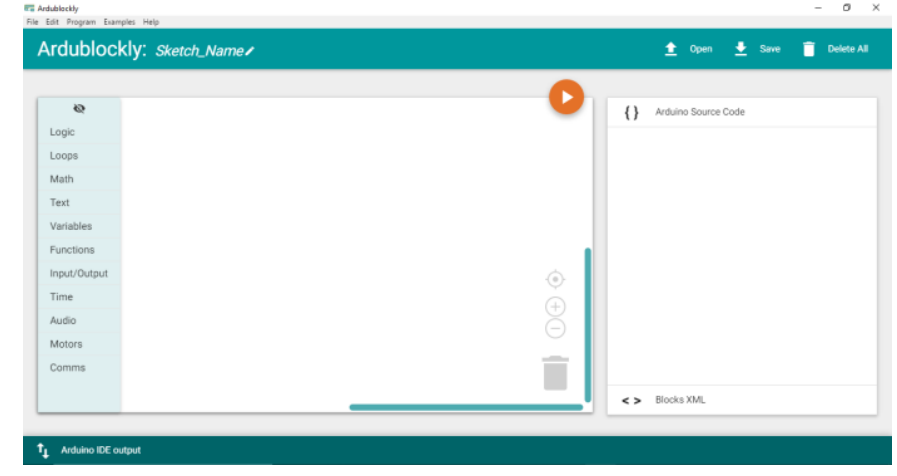

Fonte: https://github.com/carlosperate/ardublockly

\subsection{Aulas de Ciências: integrando computadores as novas linguagens}

A utilização conjunta de computadores e novas linguagens nas aulas de Ciências contribui significativamente no ensino-aprendizagem da criança, e segundo Araújo(2016), constitui uma temática relevante que agrega novas perspectivas que alguns estudos internacionais atuais têm apontado sobre as diversas formas de uso do computador e o conjunto das interfaces da tecnologia educativa na Educação em Ciências.

Segundo Araújo (2016), a integração das tecnologias dentro do espaço escolar propicia e amplia os processos de comunicação entre professores e alunos, renovando desta maneira os processos educativos de conteúdos científicos, fortalecendo desta maneira o contexto educacional.

\subsection{A robótica Pedagógica e o Ensino de Ciências}

A robótica Pedagógica, também conhecida como Robótica Educacional, têm fundamentos na Teoria Construcionista de Seymour Papert, que une a Teoria Construtivista de Jean Piaget - com quem trabalhou entre 1958 e 1963 na Universidade de Geneva, Suíça - ao uso do computador na educação(LACERDA QUEIROZ et al., 2016).

Seymour Papert, para a elaboração de teoria, aprofundou-se na Teoria de Piaget e Vygotsky, no entanto afastou-se da Psicologia do Desenvolvimento, aproximando para intervenção pedagógica mais prática(SILVEIRA, 2012).

\section{APRESENTAÇÃO E ANÁLISE DOS DADOS}

O objeto de estudos que fundamentou este artigo foi a análise dos participantes do projeto de Robótica Pedagógica, o Projeto consistiu em aplicar a robótica pedagógica como instrumento educacional para o Ensino de Ciências na Escola Municipal Major Cornélio Peixoto no Santo Antônio do Tauá. A escola foi escolhida por apresentar um baixo índice do IDEB, e nos dados da plataforma QEDU, em relação aprendizagem, com base nos resultados da Prova Brasil 2017, é possível calcular a proporção de alunos com aprendizado adequado à sua etapa escolar; (Português, $9^{\circ}$ ano) apenas 
32 (8\%) dos 372 alunos, demonstraram o aprendizado adequado, (Matemática, 90 ano) , 5 (1\%) dos 372 alunos, demonstraram o aprendizado adequado.

Os discentes da escola que participaram desta pesquisa, assinaram o termo de livre esclarecimento e consentimento para as divulgações de informações, dados e imagens contidas neste artigo, ou seja, os responsáveis dos alunos.

\section{RESULTADOS E DISCUSSÕES}

Em um dos questionamentos da pesquisa, foi realizada a seguinte indagação aos participantes: "Na sua opinião, as atividades de Robótica Pedagógica contribuíram para uma melhor compreensão dos conceitos da Física explicados no livro didático?". Conforme o Gráfico 1, observa-se que:

Gráfico 1 - Robótica Pedagógica contribui?

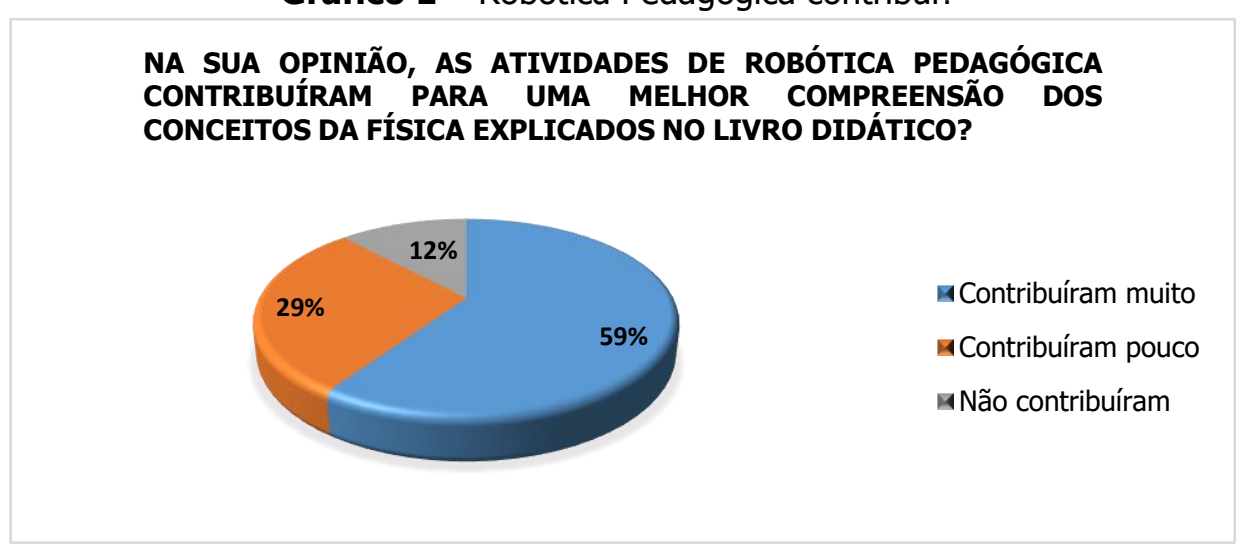

Fonte: o Autor

Observa-se que a maioria dos participantes, ou seja, 59\% afirmam que as atividades de Robótica Pedagógica contribuíram para uma melhor compreensão dos conceitos da Física explicados no livro didático, isso deu-se pelas ações do Projeto, que buscou trabalhar os conceitos estudados em sala de aula conforme Base Nacional Comum Curricular - BNCC, conforme detalhado na Tabela 1 abaixo:

Tabela 1 - Projeto - BNCC - Robótica Pedagógica

\begin{tabular}{|l|l|l|}
\hline \multicolumn{1}{|c|}{ Nome do Projeto } & $\begin{array}{l}\text { Área de Conhecimento: } \\
\text { Ciências da Natureza } \\
\text { Ciências (BNCC) }\end{array}$ & Robótica Pedagógica \\
\hline $\begin{array}{l}\text { Ligação de LED (Diodo } \\
\text { Emissor de Luz) }\end{array}$ & $\begin{array}{l}\text { Matéria e energia: Fontes e } \\
\text { tipos de energia; }\end{array}$ & $\begin{array}{r}\text { Tensão Contínua, } \\
\text { polarização, Fontes de } \\
\text { energia (pilhas, baterias, } \\
\text { energia elétrica e etc.) } \\
\text { Uso de multímetro. }\end{array}$ \\
\hline $\begin{array}{l}\text { Ligação de motores } \\
\text { Ligações de resistores em em paralelo }\end{array}$ & $\begin{array}{l}\text { Transformação de energia } \\
\text { (Elétrica em Mecânica) }\end{array}$ & \multicolumn{1}{|c|}{ Circuitos elétricos } \\
\hline
\end{tabular}

Fonte: Autor (2019)

O projeto também se utiliza dos princípios que norteiam as Olimpíadas Brasileiras de robótica (OBR), "a robótica como uma ferramenta para ajudar a aprender", os alunos foram submetidos a uma 
avaliação que contemplava as seguintes áreas do conhecimento, pois são cobradas nas avaliações externas (OBR):

- Ciências da Natureza Ciências, Matéria e energia: Fontes e tipos de energia; Transformação de energia; Cálculo de consumo de energia elétrica; Circuitos elétricos; Transformações químicas; Estrutura da matéria. Vida e evolução: Sistema reprodutivo; Hereditariedade; Biodiversidade. Terra e Universo: Universo, Sistema Solar, Terra e Lua; Clima; Ordem de grandeza astronômica; Evolução estelar.

- Além de conhecimentos sobre Robótica (Conteúdo específico), Códigos: construção e interpretação de códigos de programação mais elaborados. Sensores: identificação, princípio de funcionamento e aplicação de sensores básicos (encoder, sensor de toque, sensor de luz, sensor de cor, sonar, câmera, etc.). Atuadores: identificação e aplicação de atuadores básicos (motor de passo, motor CC, atuador pneumático, etc.). Elementos de mecânica: identificação e aplicação de elementos de mecânica básicos (rodas, engrenagens, alavancas, etc.).

Ainda na análise de resultados do Projeto Robótica Pedagógica, 100\% dos alunos associaram as atividades de robótica com as disciplinas ministradas por professores específicos, fortalecendo deste modo a interdisciplinaridade, ou seja, a Robótica Pedagógica é um elo de comunicação entre todas as disciplinas do Ensino fundamental anos finais, abaixo (tabela 2) demonstramos uma questão:

Tabela 2 - Questão

\section{Projeto 1 - Circuito LED Piscante}

Um estudante do município de Santo Antônio do Tauá, no nordeste paraense, está aprendendo a programar um Arduino por meio do Ardublockly para utilizar na árvore de natal. Para treinar, fez um programa para um LED na porta 13 piscar com uma frequência de 5 Hertz. Ele está confuso e não sabe se sua lógica está correta. Ajude-o a encontrar o código correto.

a)

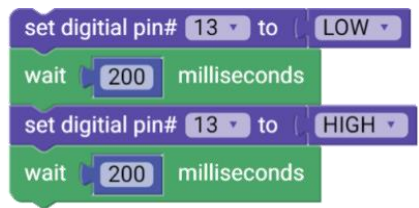

d)

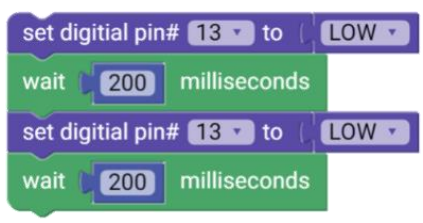

b)

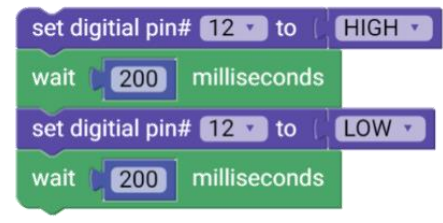

c)

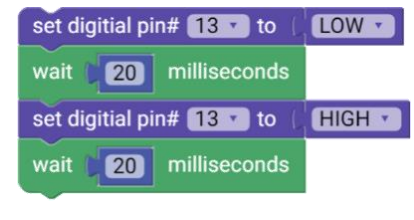

e)

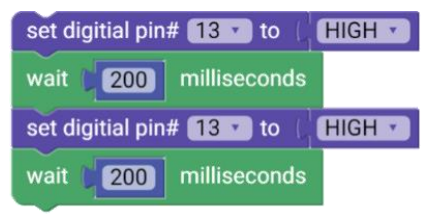

Fonte: o Autor

\section{CONSIDERAÇÕES FINAIS}

Neste artigo foi apresentado o Projeto Robótica Pedagógica à qual está sendo realizado para alunos do ensino fundamental, o resgaste do Programa PROUCA, em parceria como Laboratório de Engenhocas da UFPA, por meio de programa de Extensão, no intuito de dinamizar a educação, 
transformando uma aula monótona, em uma aula mais atrativa a fim de contemplar o pleno desenvolvimento do aluno.

A Robótica Pedagógica além de despertar o desenvolvimento pleno do discente, ainda propicia uma atividade única e dinâmica, e até mesmo lúdica, permitindo a sua construção cultural e, enquanto cidadão, torna-o autônomo, independente e responsável.

O professor, se apropriando da teoria Construcionista, torna-se um facilitador dessa metodologia inovadora, sendo capaz de auxiliar e na concretização de novos conhecimentos para os alunos. Temos a plena certeza que a Robótica Pedagógica é uma opção interessante como instrumento didática pedagógico no processo do ensino-aprendizagem para a disciplina de ciências, sendo uma proposta educativa que vem ao encontro das teorias e visões dos mais prestigiados educadores da atualidade.

\section{REFERÊNCIAS}

ARAÚJO, A. P. F. DE. PROUCA: Uma Análise Sobre a Inclusão Digital e as Práticas de Ensino de Ciências em Manaus. MANAUS: [s.n.].

ARDUBLOCKLY - Editor de Programação Visual para o Arduino. Disponível em:<https://ardublockly.embeddedlog.com/index.html/>/>. Acesso em: 25 de maio de 2019.

BRASIL. MINISTÉRIO DA EDUCAÇÃO. SECRETARIA DE EDUCAÇÃO BÁSICA. Texto Final da BNCC, 2017. Disponível em: http://basenacionalcomum.mec.gov.br/download-da-bncc. Acesso em: 15 abril de 2019.

CAMPOS, B. S.; FERNANDES, S. A.; RAGNI, A. C. P. B.; SOUZA, N. F. Física para crianças: abordando conceitos físicos a partir de situações-problema. Revista Brasileira de Ensino de Física, v. 34, 2012.

DE OliveirA, D. G. et al. PROJeto de EXTensão: A ROBÓticA PEDAGógica no ensino FUNDAMENTAL VISANDO A ENGENHARIA. [s.l: s.n.]. Disponível em: <http://www.abenge.org.br/cobenge/arquivos/3/anais/anais/161257.pdf>. Acesso em: 8 maio. 2019.

DE OLIVEIRA, D. G.; FONSECA, W. D. S. Robótica Pedagógica, uma forma diferenciada para o ensino de Ciências na região Amazônica. Revista de Estudos e Pesquisas sobre Ensino Tecnológico (EDUCITEC), v. 4, n. 09, p. 278-289, 26 dez. 2018.

LACERDA QUEIROZ, R. et al. DuinoBlocks4Kids: Um ambiente de programação em blocos para o ensino de conceitos básicos de programação a crianças do Ensino Fundamental I por meio da Robótica Educacional. Anais do CSBC, p. 2086-2095, 2016.

ROSA, H. V. Tecnologias digitais e educação : os dispositivos móveis nas políticas públicas de inserção das tecnologias na escola. [s.l: s.n.].

SILVEIRA, J. DE A. Themis Revista da ESMEC. [s.l: s.n.]. v. 10

SOUZA, A. R. DE et al. A placa Arduino: uma opção de baixo custo para experiências de física assistidas pelo PC. Revista Brasileira de Ensino de Física, v. 33, n. 1, p. 01-05, 2011.

TOLEDO, P. B. F.; ALBUQUERQUE, R. A. F.; MAGALHÃES, À. R. DE. O Comportamento da Geração Z e a Influencia nas Atitudes dos Professores. IX SEGeT 2012, 2012. 